\title{
Emergency polytetrafluoroethylene-covered stent implantation to treat right coronary artery perforation during percutaneous coronary intervention
}

\author{
Hikmet Yorgun ${ }^{1}$, Ugur Canpolat ${ }^{2}$, Kudret Aytemir' ${ }^{2}$, Ali Oto ${ }^{2}$ \\ ${ }^{1}$ Elbistan State Hospital, Kahramanmaras, Turkey \\ ${ }^{2}$ Department of Cardiology, Hacettepe University Faculty of Medicine, Ankara, Turkey
}

\begin{abstract}
Coronary artery perforations are life-threatening complications with a poor outcome. Historically, if the perforation was not controlled using conservative methods such as prolonged balloon inflation and protamine administration, emergency cardiac surgery has been performed. However, several percutaneous methods including covered stents and embolization materials have emerged as therapeutic options to manage coronary perforations. We report a case of right coronary artery perforation after high pressure stent post-dilatation that was successfully sealed with a polytetrafluoroethylene-covered stent. (Cardiol J 2012; 19, 6: 639-642)
\end{abstract}

Key words: percutaneous intervention, perforation, covered stent

\section{Introduction}

Coronary artery perforation (CAP) is a rare but life-threatening complication of percutaneous coronary interventions (PCI) $[1,2]$. The incidence of $\mathrm{CAP}$ has been reported as $0.2-0.8 \%$ among all coronary interventions [3]. Because CAPs may result in the development of cardiac tamponade and myocardial infarction, emergency coronary artery bypass grafting is the standard therapeutic option in cases of uncontrollable bleeding. However balloon inflation, covered stent implantation or embolization with embolic materials have emerged as important percutaneous therapeutic methods.

Stents covered with polytetrafluoroethylene (PTFE) have emerged as an important therapeutic option in the percutaneous sealing of CAPs. In this report, we describe a case of emergency life-saving covered stent implantion in a patient with uncontrollable perforation of the right coronary artery (RCA) during PCI.

\section{Case report}

A 53 year-old man presented with typical chest pain radiating to his left shoulder and arm. Past medical history was free of any cardiovascular disease. Among cardiovascular risk factors, he had medically controlled dyslipidemia and hypertension. The patient underwent elective coronary angiography which showed critical stenosis in the distal segment of RCA (Fig. 1A) before crux and non-significant lesions in left coronary system. PCI with stenting was planned for the patient during the same session. A clopidogrel loading dose of $600 \mathrm{mg}$ with $10,000 \mathrm{IU}$ of intravenous unfractioned heparin were given prior to procedure. A 6 Fr guiding catheter was advanced at the level of the root of the aorta after routine right femoral access. The RCA lesion was initially crossed with a 0.014 soft guidewire (Asahi Intecc, Aichi, Japan) and a $3.0 \mathrm{~mm} \times 25 \mathrm{~mm}$ bare metal stent (Multilink Zeta, Abbott Vascular, Ireland) was implanted to the lesion with a maximum

Address for correspondence: Ugur Canpolat, MD, Department of Cardiology, Hacettepe University Faculty of Medicine, Sihhiye, Ankara, P.O. 06100, Turkey, tel: +90 31230517 80, fax: +90 312311 40 58, e-mail: dru_canpolat@yahoo.com 


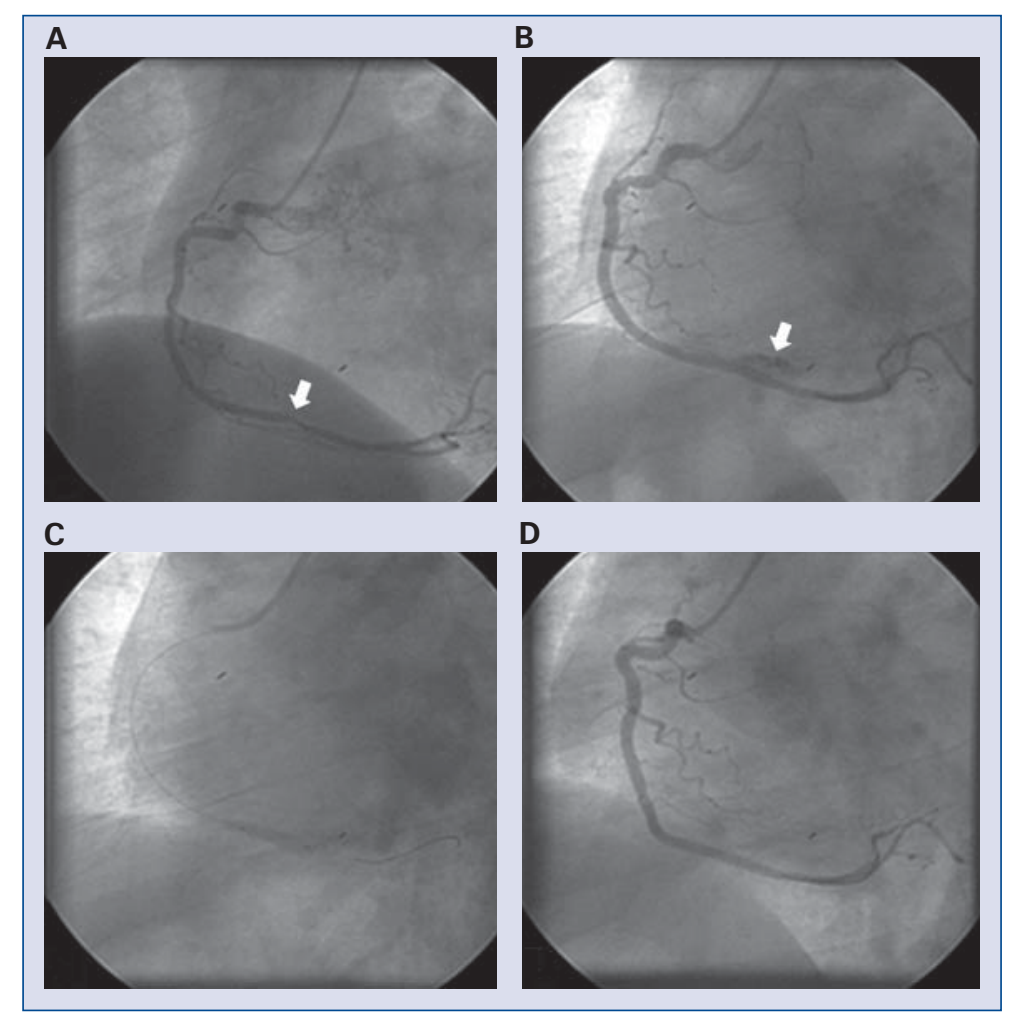

Figure 1. Coronary angiogram showing critical stenosis in the distal segment of right coronary artery (RCA) before crux (arrow) (A). Angiographic view after stent implantation revealed contrast extravasation to pericardium from the stented segment of RCA (arrow) (B). Balloon inflation with prolonged duration was performed (C). Because of the unsuccessful results with conventional methods, polytetrafluoroethylene covered stent implantation was performed to the perforated segment of the RCA (D).

12 atm (nominal pressure: 8 atm; rated burst pressure: $16 \mathrm{~atm}$ ) pressure. Because of the residual lesion, a maximum $18 \mathrm{~atm}$ pressure was reached with the stent balloon. Selective coronary angiography revealed contrast extravasation to pericardium from the stented segment of the RCA (Ellis grade III CAP) (Fig. 1B). Echocardiography confirmed the presence of pericardial effusion without cardiac tamponade. The hemodynamic status of the patient was stable. Intravenous protamine was given, with prolonged inflation of the balloon at the site of the rupture (Fig. 1C). It was decided to perform a covered stent implantation due to extravasation of the contrast medium after control injections despite conservative measures. A $3.0 \mathrm{~mm} \times 19.0 \mathrm{~mm}$ JoStent (Abbott Vascular Devices, Abbott Park, IL, USA) was deployed to the perforation site successfully and control injections revealed no extravasation (Fig. 1D). The patient recovered successfully and was discharged from hospital three days later with mild pericardial effusion which had disappeared at one month control. Dual acetylsalicylic acid and clopidogrel medication was given for six months. At a three year control visit, coronary angiography revealed a patent covered stent in the RCA without restenosis (Fig. 2A, B).

\section{Discussion}

Percutaneous coronary intervention has become a leading therapeutic treatment option for coronary artery disease [4]. With the advance of new technologies, percutaneous intervention to complex coronary lesions has increased in recent years. CAP is a rare but potentially lethal complication of coronary interventions. In a meta analysis of 16 studies by Shimony et al. [5] involving 197,061 PCIs, the pooled incidence of CAP was $0.43 \%$ (95\% CI $0.35-0.52 \%$ ). Traditionally, bypass surgery has been the preferred treatment for uncontrollable perforation; however with the development of several percutaneous methods including covered stents or embolic materials, endovascular vessel sealing has emerged as a first line treatment option.

Several patient-related risk factors have been found to be associated with the development of 


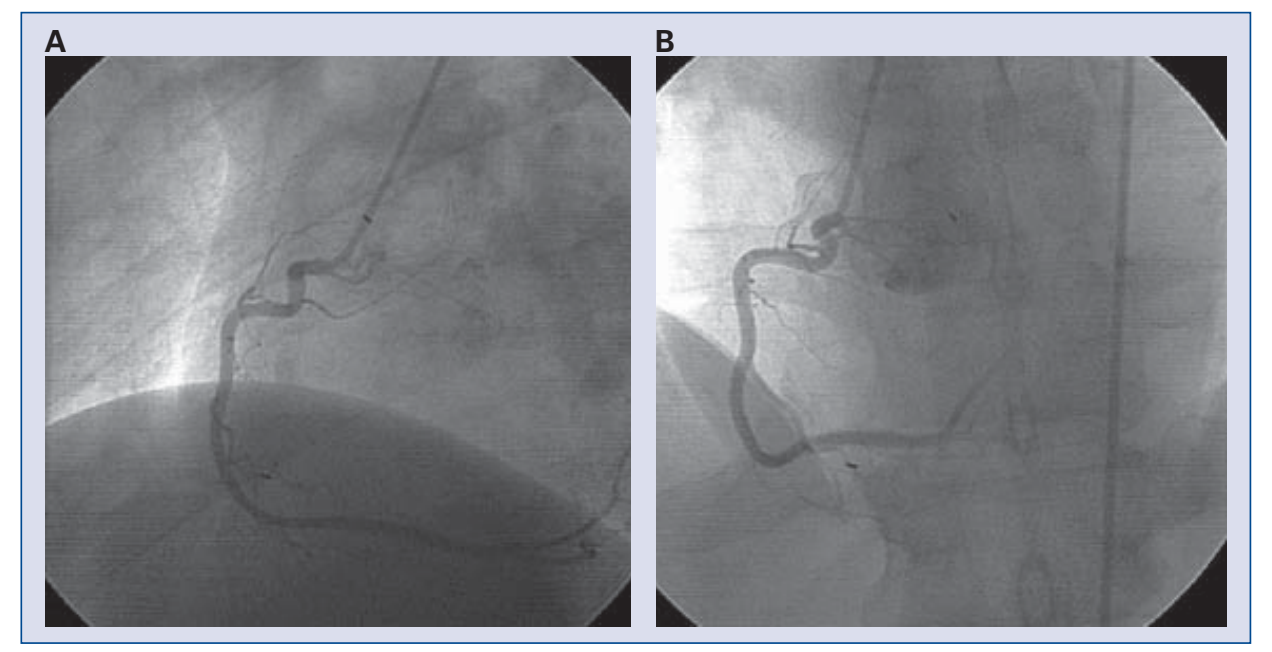

Figure 2. Coronary angiography at three year follow-up showing a patent covered stent in distal segment of right coronary artery at left $(\mathbf{A})$ and right $(\mathbf{B})$ oblique views, with no restenosis.

CAP, including older age, female gender, hypertension, history of heart failure, lower creatinine clearance and prior use of antithrombotic agents [6-8]. Also important in the development of CAP are several angiographic factors including coronary artery calcification, lesion tortuosity, and type $\mathrm{C}$ lesions and lesion related factors including use of hydrophilic guidewires, atherectomy devices, intracoronary ultrasound and stent post-dilatation with high pressure. In our patient, hypertension was the only patient-related risk factor for the development of CAP. However, the coronary lesion in the distal segment of the RCA was not a complex lesion. In our patient, CAP had developed after high pressure stent post-dilatation, which is a risk factor for CAP. Initially, we inflated an angioplasty balloon at the level of the perforation to seal the leak. Despite balloon inflation and intravenous protamine, contrast medium leaked into the pericardial cavity in subsequent injections. The hemodynamic status of the patient was stable and so we did not perform pericardiocentesis. Given the unsuccessful results with balloon inflations, we decided to perform PTFE covered stent implantation to the lesion as an alternative to surgery, and this sealed the perforation successfully.

Because of the rarity of CAPs, data was obtained from case reports or small case series. The management of CAPs is mainly based on the experience of high volume centers using different methods. PTFE is an inert and biocompatible polymer composed of carbon chains saturated with fluorine. The JoStent GraftmasterVR (Abbott Vascular, Redwood City, CA, USA) is a balloon-expandable de- vice with a single layer of PTFE sandwiched between two coaxial 316-L stainless steel, slottedtube, balloon-expandable stents [9]. Several case reports have been published about covered stent treatment of coronary perforation [10-13]. In a retrospective study with PTFE covered stents, Briguari et al. [14] reported an improvement in inhospital need for emergency cardiac surgery, cardiac tamponade and in-hospital major adverse cardiac events. However, in a previous single center retrospective study, Stankovic et al. [15] reported that covered stents reduced major adverse cardiac events only in Ellis type 3 perforations, but not type 2 perforations.

In our patient, coronary angiography three years after covered stent implantation showed that the PTFE covered stent was patent without restenosis. The main concerns regarding PTFE covered stents are stent thrombosis and in-stent restenosis. Initial studies have reported a rate of $33 \%$ for stent thrombosis at six months and $50 \%$ in-stent restenosis used for coronary perforations $[16,17]$. The proposed mechanism for those complications was delayed endothelization created by the membrane by PTFE. However, the first generation stents were not entirely covered with PTFE membrane, which was modified thereafter. Additionally, thienopyridine usage was for only 2-4 weeks in those studies. In our patient, acetylsalicylic acid and clopidogrel medication was prolonged to six months and no thrombotic event was observed. Furthermore, the prognosis of our patient was excellent, and control coronary angiography revealed a patent coronary stent three years after PTFE covered stent implantation. 
In conclusion, despite the controversy surrounding the optimal management of CAPs, covered stent is an important therapeutic option. Our case illustrates that percutaneous seal of a CAP with a PTFE covered stent is an effective method, with good mid-term follow-up results. Further improvements in the design of covered stents may improve outcomes and extend the use of PTFE covered stents for indications other than sealing perforations.

\section{Conflict of interest: none declared}

\section{References}

1. Ellis SG, Ajluni S, Arnold AZ et al. Increased coronary perforation in the new device era. Incidence, classification, management, and outcome. Circulation, 1994; 90: 2725-2730.

2. Ajluni SC, Glazier S, Blankenship L, O’Neill WW, Safian RD. Perforations after percutaneous coronary interventions: Clinical, angiographic, and therapeutic observations. Cathet Cardiovasc Diagn, 1994; 32: 206-212.

3. Javaid A, Buch AN, Satler LF et al. Management and outcomes of coronary artery perforation during percutaneous coronary intervention. Am J Cardiol, 2006; 98: 911-914.

4. Katritsis DG, Meier B. Percutaneous coronary intervention for stable coronary artery disease. J Am Coll Cardiol, 2008; 52: 889-893 .

5. Shimony A, Joseph L, Mottillo S, Eisenberg MJ. Coronary artery perforation during percutaneous coronary intervention: A systematic review and meta-analysis. Can J Cardiol, 2011 [Epub ahead of print].

6. Doll JA, Nikolsky E, Stone GW et al. Outcomes of patients with coronary artery perforation complicating percutaneous coronary intervention and correlations with the type of adjunctive antithrombotic therapy: Pooled analysis from REPLACE-2, ACUITY, and HORIZONS-AMI trials. J Interv Cardiol, 2009; 22: 453-459.
7. Shimony A, Zahger D, Van Straten M et al. Incidence, risk factors, management and outcomes of coronary artery perforation during percutaneous coronary intervention. Am J Cardiol, 2009; 104: 1674-1677.

8. Dippel EJ, Kereiakes DJ, Tramuta DA et al. Coronary perforation during percutaneous coronary intervention in the era of abciximab platelet glycoprotein IIb/IIIa blockade: An algorithm for percutaneous management. Catheter Cardiovasc Interv, 2001; 52: 279-286.

9. Romaguera R, Waksman R. Covered stents for coronary perforations: Is there enough evidence? Catheter Cardiovasc Interv, 2011; 78: 246-253.

10. Yilmaz H, Demir I, Sancaktar O, Basarici I. Successful management of osteal perforation of left anterior descending artery with coated stent. Int J Cardiol, 2003; 88: 293-296.

11. Veselka J, Tesar D, Honek T, Burkert J. Treatment of recurrent coronary rupture by implantation of three coronary stent-grafts. Int J Cardiovasc Intervent, 2003; 5: 88-91.

12. Silver KH, Bauman WB, Berkovitz KE. Dual-catheter covered stenting: A novel approach to the treatment of large coronary artery perforations. J Invasive Cardiol, 2003; 15: 348-350.

13. Lee $\mathrm{CH}$, Wong PS, Tan HC, Lim YT. Free coronary perforation after X-Sizer thrombectomy: Experience of two cases and review of the literature. J Invasive Cardiol, 2005; 17: 445-448.

14. Briguori C, Nishida T, Anzuini A, Di Mario C, Grube E, Colombo A. Emergency polytetrafluoroethylene-covered stent implantation to treat coronary ruptures. Circulation, 2000; 102: 3028-3031 .

15. Stankovic G, Orlic D, Corvaja N et al. Incidence, predictors, in-hospital, and late outcomes of coronary artery perforations. Am J Cardiol, 2004; 93: 213-216.

16. Elsner M, Auch-Schwelk W, Britten M, Walter DH, Schachinger V, Zeiher AM. Coronary stent grafts covered by a polytetrafluoroethylene membrane. Am J Cardiol, 1999; 84: 335-338.

17. Gercken U, Lansky AJ, Buellesfeld L et al. Results of the Jostent coronary stent graft implantation in various clinical settings: Procedural and follow-up results. Catheter Cardiovasc Interv, 2002; 56: 353-360. 